\title{
Co-culture induces mesenchymal stem cell differentiation and modulation of the degenerate
} human nucleus pulposus cell phenotype

Aims: While mesenchymal stem cell (MSC)-based therapies for repair of the degenerate intervertebral disc (IVD) have been proposed, the interaction of MSCs with cells of the degenerate IVD has not been fully investigated. Therefore, it is unclear whether implanted MSCs would differentiate into nucleus pulposus (NP) cells and/or stimulate endogenous NP cells. Here, we investigate the differences in interaction between human MSCs and NP cells from both nondegenerate and degenerate discs during in vitro co-culture with direct cell-cell contact. Materials \& methods: Human bone marrow-derived MSCs (labeled with CFDA) were co-cultured with direct cell-cell contact in monolayer with NP cells obtained from nondegenerate or degenerate human NP tissue from lumbar IVDs at 50:50 ratios for 7 days. Differentiation of MSCs and changes of matrix-associated genes in NP cells were assessed by quantitative real-time PCR. Results: MSCs differentiated to an NP-like phenotype following direct co-culture with both nondegenerate and degenerate NP, as shown by a significant upregulation of SOX9, type VI collagen, aggrecan and versican gene expression together with a simultaneous upregulation of CDMP-1, TGF- $\beta 1$, IGF- 1 and CTGF. Direct co-culture of normal NP cells with MSCs had no effect on the phenotype of normal NP cells, while co-culture with degenerate NP cells resulted in enhanced matrix gene expression in degenerate NP cells, accompanied by increases in both TGF- $\beta$ and CDMP-1 gene expression. Conclusion: Importantly for MSC-based therapies for repair of the degenerate IVD, these data suggest that cellular interactions between MSCs and degenerate NP cells may both stimulate MSC differentiation to an NP-like phenotype and also stimulate the endogenous NP cell population to regain a nondegenerate phenotype and consequently enhance matrix synthesis for self-repair.

\section{KEYWORDS: co-culture intervertebral disc regeneration mesenchymal stem cells nucleus pulposus}

Most people in western countries will suffer from low back pain at some point in their lives [1] and as such it is recognized as a frequent and increasing health problem. Although low back pain is a multifactorial condition, one of the main causes is thought to be degeneration of the intervertebral disc (IVD) [2,3].

The IVD consists of two main components: the outer annulus fibrosus (AF) and the inner central core, and the nucleus pulposus (NP). The NP is, in contrast to the AF, highly hydrated and gelatinous. Cells of the NP are chondrocyte-like and phenotypically distinct from AF cells. NP cells synthesize typical cartilage components such as SOX9, type II and VI collagen, aggrecan and versican [4-6]. The IVD possesses only a sparse vascularization around its periphery and thus has a poor capacity for self-repair. During disc degeneration, NP cells decrease in number [7] and the remaining cells show an altered phenotype resulting in an increased breakdown of extracellular matrix and altered matrix synthesis $[8,9]$.

To date, current treatments for low back pain are mainly restricted to targeting the symptoms rather than the underlying aberrant cell biology, even though it is now recognized that IVD degeneration is a cell-driven process originating within the NP [10]. Consequently, a range of novel biological strategies are currently being developed to treat IVD/NP degeneration, either by restoring the matrix or inhibiting matrix destruction. One approach is the direct delivery of growth factors (e.g., CDMP-1, TGF- $\beta$ and IGF-1) to the NP, which have been shown to regulate NP anabolism and stimulate matrix production [11,12]. However, the concern with this type of therapy is that there may be insufficient endogenous NP cells available to respond to the stimulus. Furthermore, it has been shown that NP cells within a degenerate disc do not respond in an appropriate manner to the exogenous growth factors [13]. Therefore, for regenerative strategies it is important to identify a suitable cell population that is able to survive within the IVD and produce an appropriate NP-like extracellular matrix.

A number of different approaches are described in the literature regarding either the use of autologous NP cells or adult mesenchymal stem cells
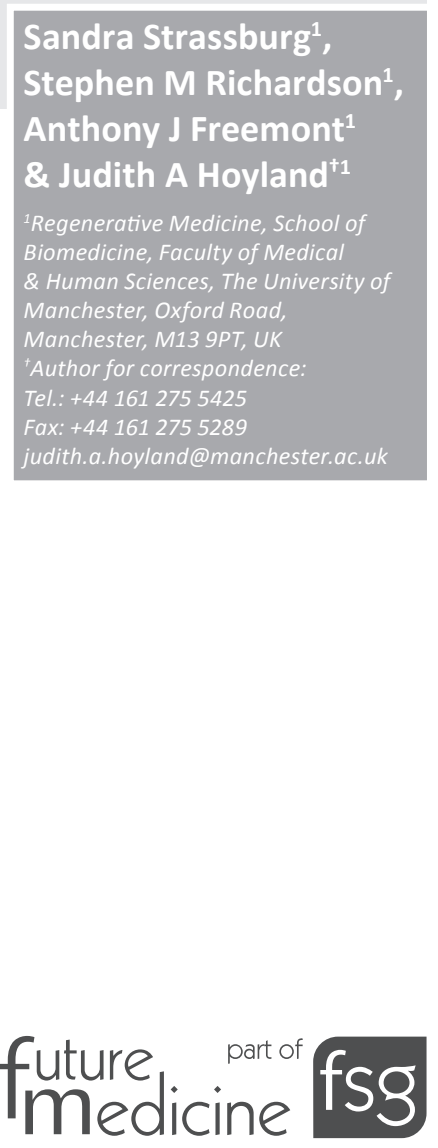
(MSCs). Recent studies in various animal models have suggested that re-implantation of autologous NP cells delays degenerative changes in the disc $[14,15]$. However, the difficulty is that the required cell number for implantation is more than can usually be obtained from a single disc biopsy. Furthermore, culturing primary NP cells for disc repair is not satisfactory due to their limited expansion capacity and further enhancement of degeneration at the donor site or initiation of degeneration if a nondegenerate donor IVD is used for cell harvest [16]. In addition, NP cells obtained from a degenerate disc show increased cellular senescence $[17,18]$ and an altered phenotype compared with healthy disc cells including upregulation of catabolic factors and degenerative enzymes, and downregulation of matrix components [19]. Therefore, the main focus for cell-based therapies is the use of MSCs to restore the normal environment of the IVD and regenerate functional NP tissue.

Mesenchymal stem cells are nonhematopoietic cells that are characterized by their multipotent differentiation potential to form tissues of mesenchymal origin [20]. They are considered to be a promising cell population since they have the ability to differentiate into chondrocyte-like cells found within the NP $[21,22]$. In addition, their differentiation potential, expansion capacity and easy availability from bone marrow aspirates make them ideal tools for the regeneration of degenerate connective tissues. In vivo animal studies, in which MSCs have been injected into the NP in order to repair the experimentally induced degenerate IVD have also demonstrated that MSCs are able to survive and proliferate within the disc environment and are able to restore normal disc structure in terms of improved disc height and accumulation of proteoglycans $[23,24]$. However, there is still controversy regarding the mechanisms by which MSCs stimulate repair when transplanted into the IVD, as it may be due to differentiation of the injected MSCs themselves and/or stimulation of native endogenous NP cells.

In order to address the question of whether MSCs differentiate to an NP-like phenotype or whether they have a stimulatory effect on native NP cells, several investigators have utilized in vitro co-culture model systems [25-31]. These studies have produced varying results depending on the culture system employed (monolayer, $3 \mathrm{D}$, indirect or direct co-culture). To date, the majority of co-culture studies have been performed in 3D cultures with minimal cell-cell contact $[27,28,30]$. However, a previous study from our laboratory has suggested that cell-cell contact is important for MSC differentiation to an NP-like phenotype since co-culture without cellular contact had no effect on the differentiation potential of MSCs [25]. Importantly, although co-culture studies have used either nondegenerate or degenerate NP cells, there are currently no studies that directly compare the differentiation potential of MSCs co-cultured with human NP cells obtained from either nondegenerate or degenerate IVDs.

Here, we investigated whether MSCs are able to differentiate to an NP-like phenotype during direct co-culture with NP cells obtained from degenerate NP tissue (compared with those cultured with NP cells derived from nondegenerate discs) and also the effect of direct co-culture on the NP cells themselves. Furthermore, we also investigated whether direct co-culture can stimulate growth factor gene expression in MSCs and NP cells, which may lead to cell differentiation and enhanced matrix gene expression within NP cells.

\section{Materials \& methods}

\section{Cell source, isolation \& maintenance}

Human MSCs were obtained from either total hip or knee replacement following local ethical committee approval and fully informed consent of patients. Bone marrow aspirates were washed once with phosphate-buffered saline (PAA Laboratories) and incubated with $250 \mu \mathrm{l}$ RosetteSep $^{\circledR}$ (StemCell Technologies Inc.) per $5 \mathrm{ml}$ bone marrow to remove unwanted blood cells. After $20 \mathrm{~min}$ incubation the sample was diluted with $5 \mathrm{ml}$ phosphate-buffered saline containing 2\% fetal calf serum (Lonza) and $1 \mathrm{mM}$ EDTA (buffer for RosetteSep) and cells were isolated using a Histopaque-1077 (Sigma) gradient centrifugation. Mononuclear cells from the interface were seeded into tissue culture plastic with $\alpha$-minimum essential medium (Gibco) supplemented with $20 \%$ fetal calf serum, $50 \mu \mathrm{g} / \mathrm{ml}$ ascorbate (Sigma), $100 \mathrm{U} / \mathrm{ml}$ penicillin, $100 \mu \mathrm{g} / \mathrm{ml}$ streptomycin and $250 \mathrm{ng}$ amphotericin B (Lonza). After 7 days the medium containing nonadherent cells was removed and adherent cells replenished with fresh medium. The multipotentiality of MSCs was assessed via differentiation along the three common mesenchymal lineages (osteogenic, adipogeneic and chondrogenic) using standard methodology (data not shown) [20]. Colonies of MSCs were cultured until subconfluence and MSCs were used for the following experiments at passage 3 . 
Nucleus pulposus cells were obtained from either nondegenerate cadaveric or surgical degenerate lumbar (L3/4-L5/S1) IVD tissue after informed consent of patients/relatives and ethical approval. NP tissue was macroscopically dissected from AF tissue and enzymatically digested at $37^{\circ} \mathrm{C}$ with $300 \mathrm{U}$ pronase (Calbiochem) followed by $0.25 \%$ collagenase type II (Gibco)/0.1\% hyaluronidase (Sigma) in serum-free medium containing antibiotics. The digested tissue/cell suspension was filtered through a $40 \mu \mathrm{m}$ cell strainer (BD Falcon) to remove remaining tissue debris. Cells were centrifuged at $400 \mathrm{~g}$ for $5 \mathrm{~min}$ and seeded onto tissue culture plastic with high glucose $(4500 \mathrm{~g} / \mathrm{l})$ Dulbecco's modified Eagle's medium (Gibco) supplemented with $10 \%$ fetal calf serum, $50 \mu \mathrm{g} / \mathrm{ml}$ ascorbate, $100 \mathrm{U} / \mathrm{ml}$ penicillin, $100 \mu \mathrm{g} / \mathrm{ml}$ streptomycin and $250 \mathrm{ng}$ amphotericin B. All cell cultures were maintained in a laminar flow hood class II and incubated at $37^{\circ} \mathrm{C}$ with $5 \% \mathrm{CO}_{2}$ and $20 \%$ oxygen in a humidified environment with a media change 2-3-times a week.

\section{Direct co-culture with subsequent cell sorting}

Prior to the direct co-culture, MSCs were fluorescently labeled with a final concentration of $10 \mu \mathrm{M}$ 5,6 caboxyfluorescein diactetate, succinimidyl ester (CFDA; Sigma) in $\alpha$-minimum essential medium for $30 \mathrm{~min}$ at $37^{\circ} \mathrm{C}$ in the dark. The reaction was halted by the addition of excess media and by washing cells with Hank's Buffered Salt Solution (HBSS; PAA Laboratories).

Direct co-cultures were performed in monolayer at 50:50 ratios $\left(12,000\right.$ cells $/ \mathrm{cm}^{2}$ in total $)$ in six-well plates (Becton Dickinson) in triplicate. Co-cultures were established between MSCs ( $\mathrm{n}=4$; two males 57 and 60 years, two females 44 and 76 years; mean age: 59 years) and cells derived from nondegenerate (histological grade 1-2 [assessed using criteria published by [4] ]; $n=4$, all male, ages 37, 46, 47 and 57 years; mean age: 47 years) and degenerate discs (histological grade 7-10 [assessed using criteria published by [4]]; $\mathrm{n}=4$, two females ages 36 and 51 years, two males 43 years; mean age: 43 years). Notably, the same MSC samples were cultured with both nondegenerate and degenerate cells. CFDA-labeled MSCs and unlabeled NP cells cultured alone served as controls and were seeded at the same density. All co-cultures and controls were analyzed after 7 days. Following co-culture, cells were separated as previously described [25]. Briefly, cells were trypsinized and washed in HBSS to remove all remaining media and serum components and then resuspended in $400 \mu \mathrm{l}$ HBSS and filtered through a $50 \mu \mathrm{m}$ filter (BD Biosciences) to remove cell clumps. Cell sorting (BD Biosciences FACS Aria high speed cell sorter with Diva 5 software) was used to analyze and separate CFDA-labeled MSCs from unlabeled NP cells after direct co-culture. Gates were set to exclude all dead cells, cell debris and cell clumps. Within the gated cell population, cells fluorescing at $530 \mathrm{~nm}$ were identified as CFDA-labeled MSCs and nonfluorescent cells as unlabeled NP cells. Fluorescent and nonfluorescent cells were sorted into $500 \mu \mathrm{l}$ Dulbecco's modified Eagle's medium in $5 \mathrm{ml}$ polypropylene tubes (BD Biosciences).

\section{RNA extractions, cDNA synthesis \& quantitative real-time PCR}

Sorted cells were pelleted at $500 \mathrm{~g}$ for $5 \mathrm{~min}$ and lysed in $1 \mathrm{ml} \mathrm{TRIzol}^{\circledR}$ (Invitrogen). Control cells were rinsed in HBSS and lysed in $1 \mathrm{ml}$ TRIzol for total RNA extraction according to the manufacturer's instructions. Briefly, chloroform was added to each sample for phase separation. Total RNA was then precipitated by addition of isopropanol and high-salt precipitation solution $(0.0038 \mathrm{M}$ sodium citrate $+1.2 \mathrm{M} \mathrm{NaCl}$ ) to the aqueous phase, followed by centrifugation. RNA pellets were washed in $75 \%$ ethanol and resuspended in RNase-free water (Invitrogen). Following precipitation, RNA was treated with DNase (Invitrogen) to ensure DNA-free RNA.

Reverse transcription was performed using Superscript II and random hexamers to generate complementary DNA (cDNA) from the isolated total RNA (all reagents from Invitrogen). RNA (500 ng) was mixed with random hexamers (250 ng) and deoxyribonucleotide triphosphates $\left(500 \mu \mathrm{M}\right.$ each), incubated at $65^{\circ} \mathrm{C}$ for $5 \mathrm{~min}$ and immediately cooled on ice. The reaction was made up to $20 \mu \mathrm{l}$ with $5 \times$ firststrand buffer, dithiothreitol $(0.1 \mathrm{M})$, RNase inhibitor (40 U) and Superscript II (200 U) and incubated at $25^{\circ} \mathrm{C}$ for $10 \mathrm{~min}$ before heating to $42^{\circ} \mathrm{C}$ for $90 \mathrm{~min}$ to synthesize cDNA. Finally, the reaction was incubated at $70^{\circ} \mathrm{C}$ for $10 \mathrm{~min}$ to inactivate the enzyme.

Quantitative real-time PCR (QRT-PCR), using an ABI Prism 7000 system (Applied BioSystems), was performed to quantify gene expression. Preoptimized primers and probes for SOX9 (forward (F): GACTTCCGCGACGTGGAC; reverse (R): 
GTTGGGCGGCAGGTACTG; probe (P): CGACGTCATCTCCAACAT), type VI collagen (F: TCAAGGAGGCTGTCAAGAACCT; R: CCTTGATGAGGCGGTCGTA; P: CACCTGGACACCCTCAGCCCTCAAGT), aggrecan (F: CCGTGTGTCCAAGGAGAAGG; R: GGGTAGTTGGGCAGTGAGAC; P: CTGATAGGCACTGTTGAC) and versican (F: TGGAAAAGTCAGCCTACCTTGTC; R: GCGGAGAAATTCACTGGTGTTG; P: TTTCAACGATGCCTACTTTGC CACCCAGT) were purchased from Applied BioSystems for TaqMan real-time PCR. Primers were also designed and optimized for CDMP-1 (F: CTGTGCGAGTTCCCATTGC; R: TGGAGTTCATCAGGGTCTGGAT), TGF- $\beta 1$ (F: CGCGCATCCTAGACCCTTT; R: CTGTGGCAGGTCGGAGAGA), IGF-1 (F: AGCCTGTCCACCCTTGAGAA; R: CCCTGGAGCCACAGAGCAT) and CTGF (F: CCCTGCATCTTCGGTGGTA; R: GGCACGTGCACTGGTACTTG) for SYBR-green QRT-PCR. GAPDH primer/ probe set was predesigned and purchased from Applied BioSystems; for SYBR-green QRT-PCR $G A P D H$ primers were designed and optimized (F: GCTGAACGGGAAGCTCACT; R: AGGTCAGGTCCACCACTGA). Reactions were performed in duplicate in 96-well plates (Applied BioSystems). Each reaction was made up to a total volume of $25 \mu \mathrm{l}$ using $2.5 \mu \mathrm{l}$ of cDNA template (25 ng). For TaqMan primer/ probe sets, cDNA was mixed with $12.5 \mu \mathrm{l}$ Universal PCR mastermix (Applied BioSystem), a final concentration of $900 \mathrm{nM}$ forward and reverse primer, $250 \mathrm{nM}$ appropriate probe and water accordingly to the final volume. For SYBR-green, cDNA was mixed with $12.5 \mu \mathrm{l}$ SYBR green mastermix (Applied BioSystem), a final concentration of $600 \mathrm{nM}$ or $900 \mathrm{nM}$ forward and reverse primer and water accordingly to the final volume. All QRT-PCR data were normalized to the housekeeping gene GAPDH using the $2^{-\Delta C \mathrm{t}}$ method [32].

\section{- Statistical analysis}

Statistical analysis was performed with GraphPad InStat software using the Mann-Whitney U-test, and significance was defined as $\mathrm{p}<0.05$.

\section{Results}

\section{Direct co-culture \& cell sorting}

Carboxyfluorescein diacetate-labeled MSCs and nondegenerate or degenerate NP cells were cocultured with direct cell-cell contact in monolayer. During the 7 days of co-culture, cells maintained their native morphological appearance and no changes in cell shape were observed microscopically.

A cell sorter was used to separate the two cell populations after co-culture. A single cell suspension was analyzed using forward and side scatter. A region (P1) was drawn in a $2 \mathrm{D}$ dot plot (Figure 1A) to exclude dead cells, cell debris and cell clumps. CFDA-negative cells (NP cells) and CFDA-positive cells (MSCs) were selected using different gates in fluorescence versus side scatter dot plot (Figure 1B). Therefore, a gate (P2) was set over CFDA-negative cells and the gate (P3) was set over CFDA-positive cells, leaving a gap between the two gates to ensure no cross contamination.

\section{Effect of direct co-culture on MSC \&} NP cell phenotype

Quantitative real-time-PCR was used to analyze the mRNA expression of the NP (chondrocytelike) marker genes (SOX9, type VI collagen, aggrecan and versican) in the sorted MSC and NP cell population after 7 days of direct co-culture with cell-cell contact.

\section{Gene expression in MSCs following direct co-culture with NP cells}

When MSCs were co-cultured with either nondegenerate or degenerate NP cells, MSCs showed a significant $(\mathrm{p}<0.05)$ increase in SOX9, type VI collagen and versican mRNA expression after 7 days of direct co-culture compared with MSCs cultured alone. Aggrecan gene expression in MSCs was significantly increased following co-culture with degenerate NP cells (Figure 2).

\section{Gene expression in NP cells following direct co-culture with MSCs}

Baseline levels of expression for SOX9, aggrecan and versican were lower in degenerate NP control cells compared with nondegenerate NP control cells, with baseline expression for type VI collagen significantly $(\mathrm{p}<0.05)$ lower in degenerate NP control cells compared with nondegenerate NP control cells (Figure 3).

Following direct co-culture with MSCs, no significant changes in gene expression were seen in nondegenerate NP cells after 7 days. In contrast to nondegenerate NP cells, degenerate NP cells showed an increase in SOX9 and significant increases in type VI collagen $(\mathrm{p}<0.001)$, aggrecan $(\mathrm{p}<0.05)$ and versican $(\mathrm{p}<0.05)$ mRNA expression after 7 days of direct co-culture with MSCs to levels seen in nondegenerate NP control cells (Figure 3). 

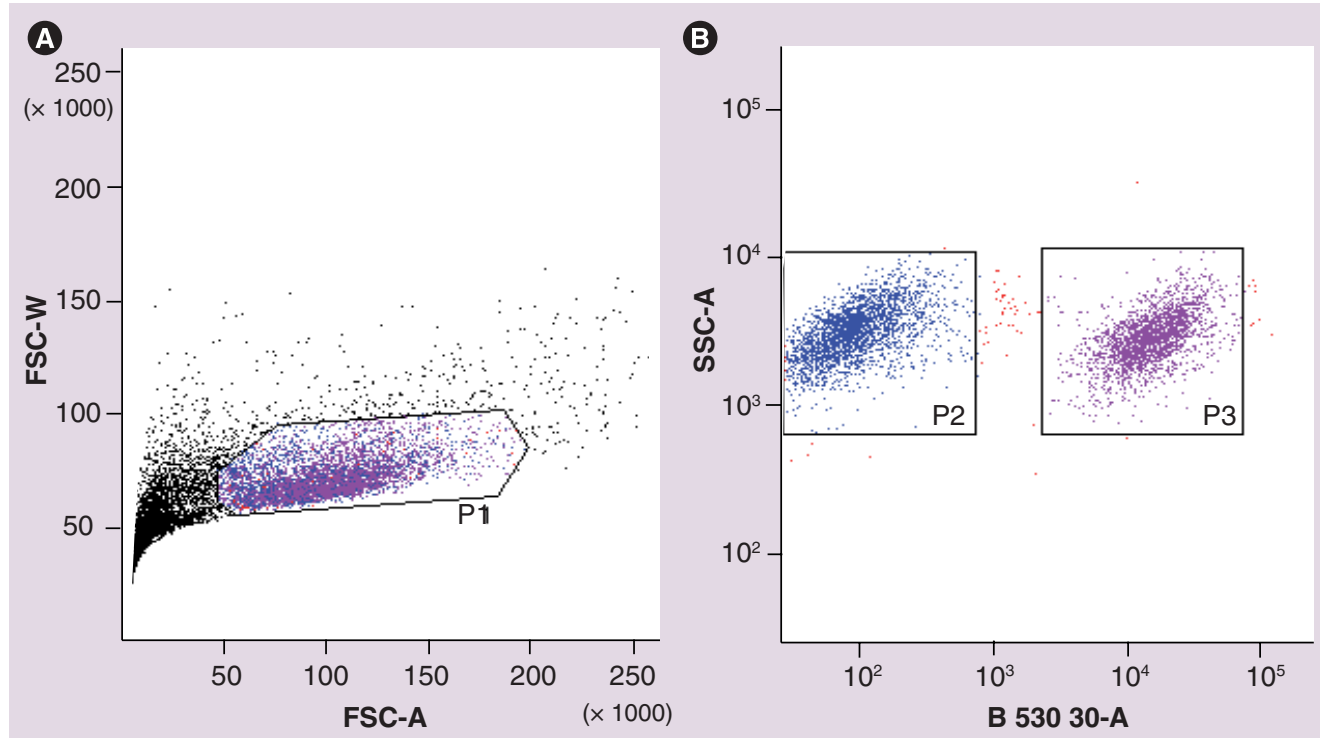

Figure 1. Example of cell sorting data for separation of carboxyfluorescein diacetatelabeled mesenchymal stem cells and unlabeled nucleus pulposus cells following direct co-culture. (A) 2D dotplot for co-cultured cells together. Gate P1 was placed around single live cells. (B) 2D dotplot showing unlabeled nucleus pulposus cells in gate P2 and carboxyfluorescein diacetate-labeled mesenchymal stem cells in gate P3 that were sorted.

FSC-A: Forward scatter - area; FSC-W: Forward scatter - width; SSC-A: Side scatter - area.

\section{Effect of direct co-culture on} growth factor gene expression in MSCs \& NP cells

Mesenchymal stem cells were either co-cultured with nondegenerate or degenerate NP cells and 7 days after direct co-culture fluorescently labeled MSCs and unlabeled NP cells were separated by cell sorting and gene expression for CDMP-1, TGF- $\beta 1, I G F-1$ and $C T G F$ assessed.

\section{Growth factor gene expression in MSCs}

Mesenchymal stem cells showed significant $(\mathrm{p}<0.05)$ increases in $C D M P-1, T G F-\beta 1$, $I G F-1$ and $C T G F$ mRNA expression after 7 days of direct co-culture with both nondegenerate or degenerate NP cells compared with the control (Figure 4). No significant difference was observed between MSCs co-cultured with nondegenerate or degenerate NP cells.

\section{Growth factor gene expression in} nondegenerate \& degenerate NP cells

No statistical differences were observed between mRNA expression levels of CDMP-1 and TGF- $\beta 1$ in nondegenerate or degenerate NP control cells (FIGUre 5). There was no effect of direct co-culture on growth factor gene expression in nondegenerate NP cells after 7 days, but degenerate NP cells showed a significant increase in CDMP-1 and TGF- $\beta 1$ after 7 days of direct co-culture with MSCs compared with controls ( $\mathrm{p}<0.05)$ (Figure 5). No changes in $I G F-1$ and $C T G F$ were observed (data not shown).

\section{Discussion}

Cell-based therapies to repair the degenerate IVD endeavor to increase the number of viable cells within the tissue capable of producing an appropriate and functional NP-like matrix. As NP cells have been shown not to be an ideal cell population for IVD repair, the main focus for cell-based therapies has been on the application of MSCs, as these have been shown to differentiate in vitro into chondrocyte-like NP cells [22]. Furthermore, in vivo studies have shown that MSCs are able to survive and proliferate within animal experimentally induced degenerate discs and that following implantation normal disc structure is restored after a specific time period $[23,24,33]$. However, in this IVD 'niche' it is not known whether injection of MSCs into the IVD leads to MSC differentiation to an NP-like phenotype and/ or whether injected MSCs promote regeneration through stimulation of resident native NP cells.

In order to delineate the effects on either cell type, several investigators have utilized coculture model systems. Here, we have further investigated the in vitro interaction between MSCs and NP cells using a direct co-culture system to verify whether MSCs differentiate 

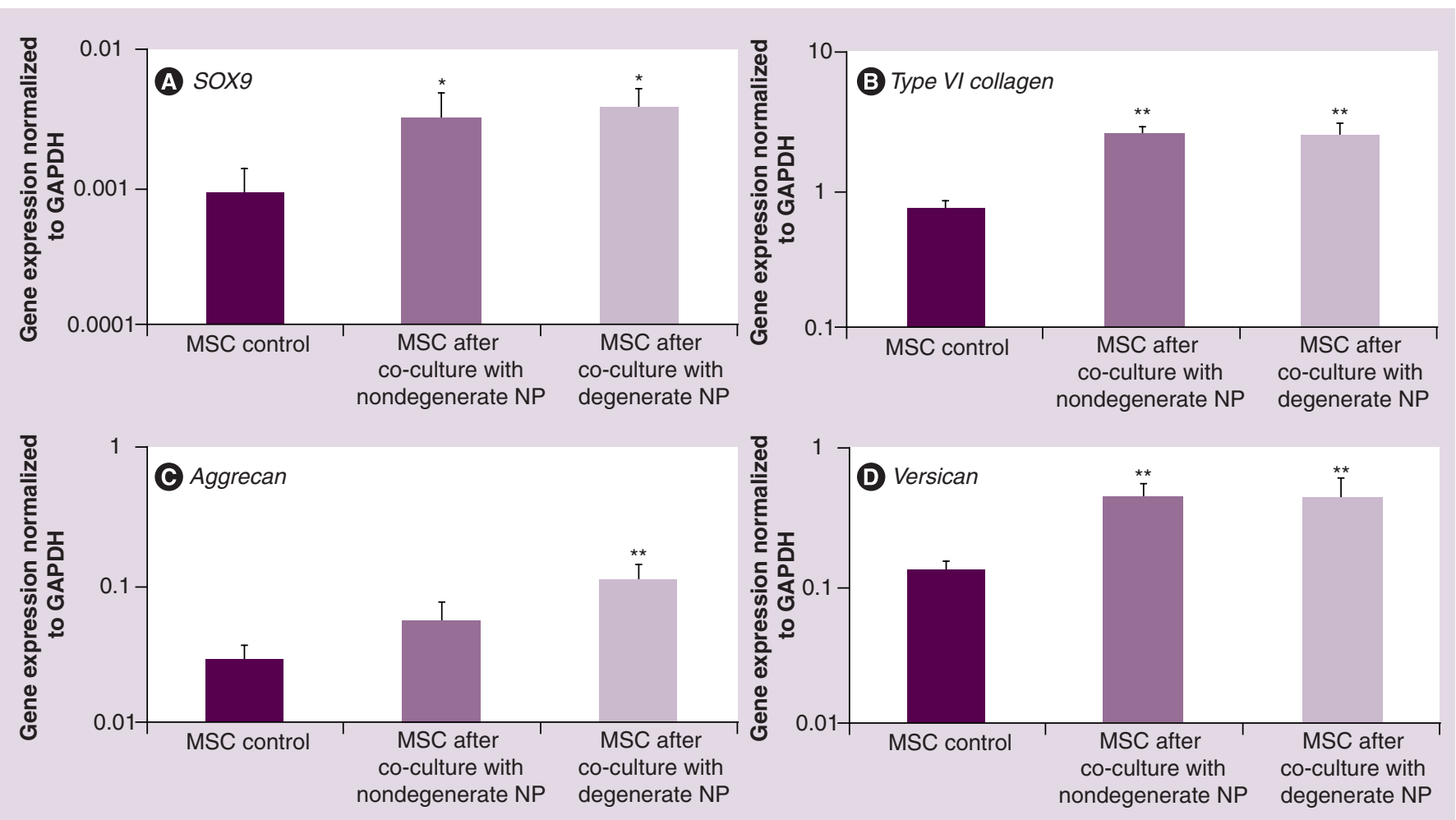

Figure 2. Relative gene expression in mesenchymal stem cells after 7 days of direct co-culture with either nondegenerate or degenerate nucleus pulposus cells. Gene expression for each sample was normalized to the housekeeping gene GAPDH and plotted on a log scale (y-axis). Data were averaged from four different patient samples and performed in triplicate. (A) SOX9, (B) type VI collagen, (C) aggrecan and (D) versican.

${ }^{*} p<0.05 ;{ }^{* *} p<0.001$.

MSC: Mesenchymal stem cell; NP: Nucleus pulposus.

to NP cells or whether they stimulate NP cells in terms of enhanced matrix gene expression. Importantly, we have shown that human MSCs acquire an NP-like phenotype after being directly co-cultured with human NP cells derived from either nondegenerate or degenerate discs. Interestingly, we have also shown that during direct co-culture, matrix-associated gene expression in degenerate NP cells was significantly increased, whereas there was no change in gene expression in cells derived from nondegenerate discs. This suggests that MSCs exert a trophic effect on degenerate NP cells, but not on nondegenerate NP cells. Associated with this was an increased mRNA expression for the growth factors CDMP-1, TGF- $\beta 1$, IGF-1 and CTGF in MSCs after direct coculture with both nondegenerate or degenerate NP cells and increased mRNA expression of CDMP-1 and TGF- $\beta 1$ in degenerate NP cells after direct co-culture with MSCs.

Although a number of different co-culture systems have been employed to induce and maintain an NP-like phenotype in MSCs, previous studies have involved the addition of growth factors or a $3 \mathrm{D}$ environment that makes the subsequent isolation and examination of cells difficult $[27,28]$. We opted to use a monolayer co-culture model system with direct cell-cell contact, since recent studies by both Richardson et al. and Yamamoto et al. demonstrated that direct cell-cell contact was vital for MSC differentiation, as indirect co-cultures (separation of MSCs and NP cells through a semi-permeable membrane) had no effect on MSC differentiation [25,26]. To the best of our knowledge, the current study is the first to directly compare the in vitro interaction between MSCs and NP cells derived from nondegenerate and degenerate discs using a direct co-culture system with cell-cell contact in monolayer.

To assess cellular differentiation during direct co-culture, we examined the genes SOX9, type VI collagen, aggrecan and versican, which have previously been shown to be expressed by NP cells [4-6]. SOX9 is the most commonly used marker of MSC differentiation to chondrocyte-like cells, such as NP cells. It is responsible for the induction of type II collagen, which is expressed later during chondrogenic differentiation. The loss of type II collagen 
expression during monolayer culture of NP cells is a classic sign of dedifferentiation and MSCs show no or low expression of both type II collagen and SOX9. Type II collagen expression in monolayer is also variable as a number of common culture media components, particularly ascorbic acid, can affect its expression. Thus, SOX9, which is considered to be a more sensitive and reliable marker of chondrogenic differentiation, was chosen as a differentiation marker in this study in preference to type II collagen. To confirm differentiation to an NP-like phenotype other NP cell marker genes were also analyzed, including type VI collagen, a pericellular protein that is expressed in NP tissue [6], and aggrecan and versican, which have also been reported as being abundantly expressed in the NP [5].

Several studies have shown that MSCs exert a trophic effect on cells in their vicinity by secretion of a variety of growth factors and cytokines that have the potential to stimulate the cells [34]. In particular, Yamamoto et al. found that cell-cell contact between MSCs and NP cells induced the secretion of growth factors (TGF- $\beta 1$, IGF-1, EGF and PDGF) into the media [26]. However, their study did not separate the two cell types after co-culture, thus making it impossible to establish whether NP cells or MSCs were responsible for the increase in growth factors in the media. Although in the current study we do not demonstrate secretion of growth factors into the medium, we have shown that MSCs significantly enhance mRNA expression for CDMP- 1 and $T G F-\beta 1$ in degenerate NP cells following direct co-culture. Furthermore, both nondegenerate and degenerate NP cells significantly increased mRNA expression for CDMP-1, TGF- $\beta 1, I G F-1$ and $C T G F$ in MSCs after direct co-culture. This increased growth factor gene expression might be the mechanism responsible for the observed MSC differentiation to an NP-like phenotype and the enhanced matrix-associated gene expression in degenerate NP cells. Recently, researchers have shown a renewed interest in identifying the effect of growth factors on MSC differentiation to a chondrogenic lineage and on the anabolic regulation of NP cells. CDMP-1 and TGF- $\beta 1$ have been reported to enhance chondrogenesis in adenovirustransduced MSCs $[35,36]$ and a combination of
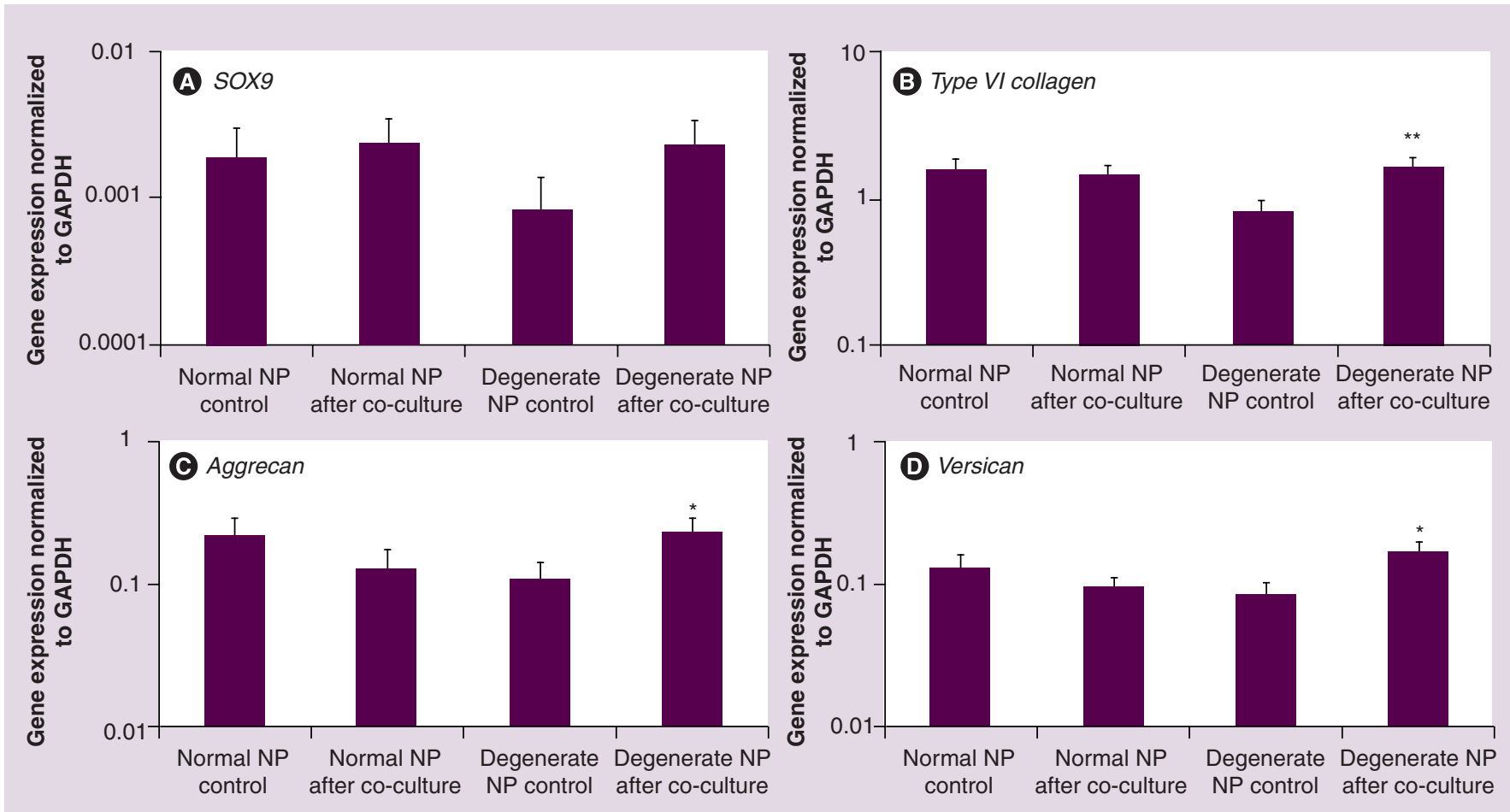

Figure 3. Relative gene expression in nondegenerate and degenerate nucleus pulposus cells after 7 days of direct co-culture with mesenchymal stem cells. Gene expression for each sample was normalized to the housekeeping gene GAPDH and plotted on a log scale (y-axis). Data were averaged from four different patient samples and performed in triplicate. (A) SOX9, (B) type VI collagen, (C) aggrecan and (D) versican.

${ }^{*} \mathrm{p}<0.05 ;{ }^{* *} \mathrm{p}<0.01$.

MSC: Mesenchymal stem cell; NP: Nucleus pulposus. 

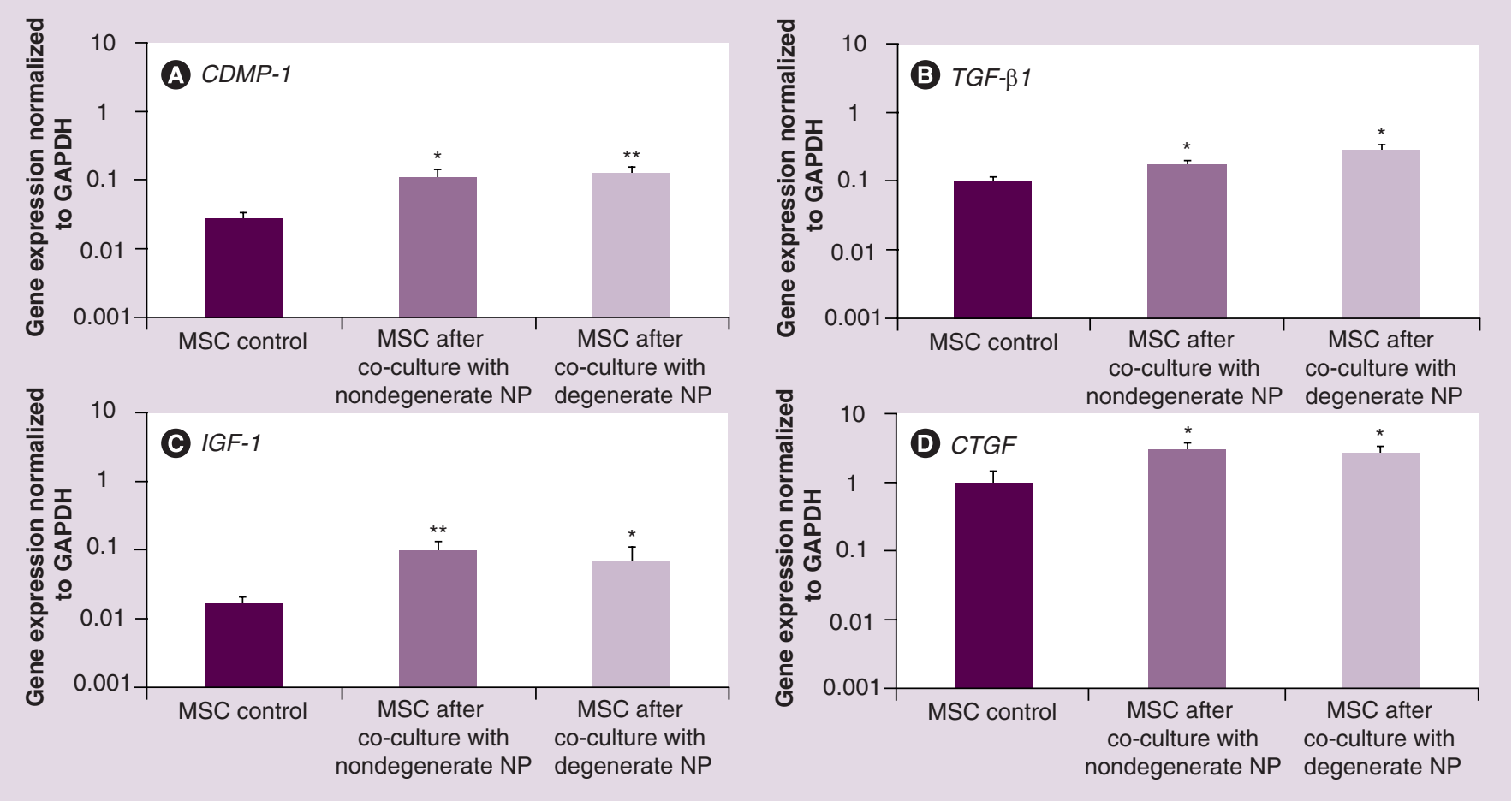

Figure 4. Relative gene expression in mesenchymal stem cells after 7 days of direct co-culture with either nondegenerate or degenerate nucleus pulposus cells. Gene expression for each sample was normalized to the housekeeping gene GAPDH and plotted on a log scale (y-axis). Data were averaged from three different patient samples and performed in triplicate. (A) CDMP-1, (B) TGF- $\beta 1$, (C) IGF-1 and (D) CTGF.

${ }^{*} \mathrm{p}<0.05 ; * * \mathrm{p}<0.0001$.

MSC: Mesenchymal stem cell; NP: Nucleus pulposus.

CDMP-1 and TGF- $\beta 1$ was more effective for the induction of chondrogenesis than either of the growth factors alone [37]. Although IGF-1 alone has not been reported to enhance chondrogenesis in MSCs, it has been shown that a combination of TGF- $\beta$ and IGF-1 synergistically induced chondrogenesis [38,39]. QRT-PCR assays have also shown that CTGF mRNA is upregulated in MSCs when they undergo chondrogenesis [40]. In addition, stimulation of disc cells with recombinant CDMP-1 in vitro resulted in upregulation of proteoglycan and collagen synthesis $[12,41]$.

For the first time, we have demonstrated that nondegenerate and degenerate NP cells behave differently during direct co-culture
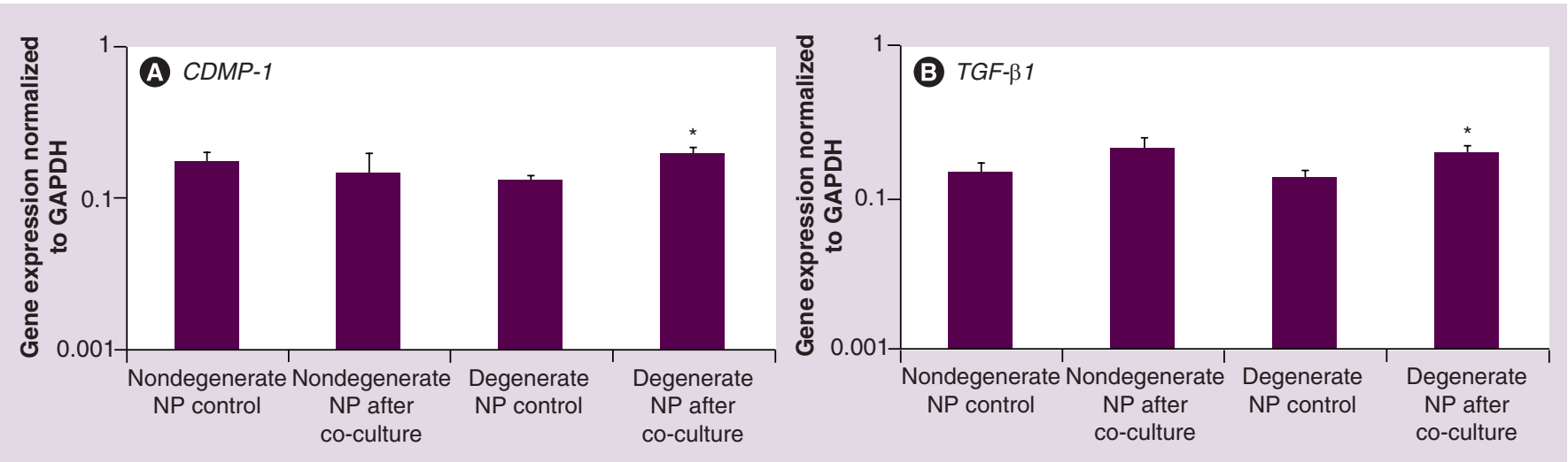

Figure 5. Relative gene expression in nondegenerate and degenerate nucleus pulposus cells after 7 days of direct co-culture with mesenchymal stem cells. Gene expression for each sample was normalized to the housekeeping gene GAPDH and plotted on a log scale (y-axis). Data were averaged from three different patient samples and performed in triplicate. (A) CDMP-1 and (B) TGF- $\beta 1$.

${ }^{*} \mathrm{p}<0.05$.

NP: Nucleus pulposus. 
with MSCs. While both nondegenerate and degenerate NP cells were able to stimulate MSC differentiation to an NP-like phenotype, MSCs were only able to stimulate degenerate NP cells to increase their matrix-associated genes to levels comparable to nondegenerate $\mathrm{NP}$ cells. This suggests that during co-culture the degenerate NP cells regain a nondegenerate phenotype and supports the idea that MSCs may behave in a trophic manner on degenerate NP cells. This finding is in agreement with the results of a study by Vadala $e t$ al., which suggested a trophic effect of degenerate NP cells on MSCs but only a modest trophic effect of MSCs on degenerate NP cells [27]. We hypothesize that this modest trophic effect may be the result of different co-culture conditions. The previous study used a direct 3D system with little cell-cell contact, while ours used a monolayer co-culture system with extensive cell-cell contact.

Recently, several in vivo studies have demonstrated that MSC implantation into the degenerate IVD leads to restoration of a disclike matrix, although the exact mechanisms are not understood $[23,24,33,42,43]$. This current study further clarifies the mechanism of the interaction between MSCs and degenerate NP cells. Our data suggest that implantation of MSCs into a degenerate disc may lead to MSC differentiation to an NP-like phenotype and simultaneous enhancement of the phenotype of degenerate NP cells to a nondegenerate NP cell phenotype. Thus, MSCs may be able to stimulate degenerate NP cells for self-repair. Furthermore, the differentiation of MSCs to an NP-like phenotype and the recovery of a nondegenerate phenotype in degenerate NP cells during direct co-culture may be explained by the increase in growth factor gene expression. Growth factors and their role in directing MSC and NP behavior in an environment resembling the degenerate IVD is therefore an aspect that requires further investigation.

\section{Conclusion}

This study demonstrates that during direct monolayer co-culture, both nondegenerate and degenerate NP cells are able to stimulate MSC differentiation to an NP-like phenotype. Interestingly, following direct co-culture with MSCs only degenerate NP cells (but not nondegenerate NP cells) show enhanced matrix gene expression to levels seen in nondegenerate NP cells. The observed MSC differentiation to an NP-like phenotype and the enhancement of matrix-associated genes in degenerate NP cells (and possible restoration to a nondegenerate NP phenotype) might be due to increased growth factor mRNA expression (CDMP-1, TGF- $\beta 1$, IGF-1 and CTGF in MSCs; CDMP-1 and $T G F-\beta 1$ in degenerate NP cells) following coculture. Thus, in terms of clinical applications for a cell-based therapy to repair the degenerate IVD, implantation of MSCs into a degenerate IVD may both stimulate MSC differentiation to an NP-like phenotype and also stimulate the endogenous NP cell population to regain a nondegenerate phenotype and consequently enhance matrix synthesis for self-repair.

Financial \& competing interests disclosure

The authors have no relevant affiliations or financial involvement with any organization or entity with a financial interest in or financial conflict with the subject matter or materials discussed in the manuscript. This includes employment, consultancies, honoraria, stock ownership or options, expert testimony, grants or patents received or pending, or royalties.

No writing assistance was utilized in the production of this manuscript.

\section{Ethical conduct of research}

The authors state that they have obtained appropriate institutional review board approval or have followed the principles outlined in the Declaration of Helsinki for all human or animal experimental investigations. In addition, for investigations involving human subjects, informed consent has been obtained from the participants involved.

\section{Executive summary}

- Nucleus pulposus (NP) cells obtained from either nondegenerate or degenerate intervertebral discs are able to stimulate mesenchymal stem cell (MSC) differentiation to an NP-like phenotype during direct co-culture.

- Co-culture of MSCs with degenerate NP cells results in increased gene expression for matrix proteins in degenerate NP cells to levels seen in nondegenerate NP cells.

- There is no effect of MSCs on normal NP cells during direct co-culture.

- Growth factor gene expression is increased in MSCs during direct co-culture with NP cells.

- Growth factor gene expression is only increased in degenerate NP cells, not normal NP cells.

- Injection of MSCs into the degenerate intervertebral disc may lead to MSC differentiation to the appropriate phenotype and stimulation of endogenous intervertebral disc cells to induce self-repair. 


\section{Bibliography}

1 Waddell G: Low back pain: a twentieth century health care enigma. Spine 21(24), 2820-2825 (1996).

2 Luoma K, Riihimaki H, Luukkonen R, Raininko R, Viikari-Juntura E, Lamminen A: Low back pain in relation to lumbar disc degeneration. Spine 25(4), 487-492 (2000).

3 Cheung KM, Karppinen J, Chan D et al.: Prevalence and pattern of lumbar magnetic resonance imaging changes in a population study of one thousand forty-three individuals. Spine 34(9), 934-940 (2009).

4 Sive JI, Baird P, Jeziorsk M, Watkins A, Hoyland JA, Freemont AJ: Expression of chondrocyte markers by cells of normal and degenerate intervertebral discs. Mol. Pathol. 55(2), 91-97 (2002).

5 Melrose J, Ghosh P, Taylor TK: A comparative analysis of the differential spatial and temporal distributions of the large (aggrecan, versican) and small (decorin, biglycan, fibromodulin) proteoglycans of the intervertebral disc. J. Anat. 198(Pt 1), 3-15 (2001).

6 Roberts S, Ayad S, Menage PJ: Immunolocalisation of type VI collagen in the intervertebral disc. Ann. Rheum. Dis. 50(11), 787-791 (1991).

7 Urban MR, Fairbank JC, Bibby SR, Urban JP: Intervertebral disc composition in neuromuscular scoliosis: changes in cell density and glycosaminoglycan concentration at the curve apex. Spine (Phila Pa 1976), 26(6), 610-617 (2001).

8 Le Maitre CL, Pockert A, Buttle DJ, Freemont AJ, Hoyland JA: Matrix synthesis and degradation in human intervertebral disc degeneration. Biochem. Soc. Trans. 35(Pt 4), 652-655 (2007).

9 Pockert AJ, Richardson SM, Le Maitre CL et al:: Modified expression of the ADAMTS enzymes and tissue inhibitor of metalloproteinases 3 during human intervertebral disc degeneration. Arthritis Rheum. 60(2), 482-491 (2009).

10 Freemont AJ, Watkins A, Le Maitre C, Jeziorska M, Hoyland JA: Current understanding of cellular and molecular events in intervertebral disc degeneration: implications for therapy. J. Pathol. 196(4), 374-379 (2002).

11 Thompson JP, Oegema TR Jr, Bradford DS: Stimulation of mature canine intervertebral disc by growth factors. Spine 16(3), 253-260 (1991).

12 Chujo T, An HS, Akeda K et al.: Effects of growth differentiation factor- 5 on the intervertebral disc - in vitro bovine study and in vivo rabbit disc degeneration model study. Spine 31(25), 2909-2917 (2006).
13 Le Maitre CL, Freemont AJ, Hoyland JA: The role of interleukin-1 in the pathogenesis of human intervertebral disc degeneration. Arthritis Res. Ther. 7(4), R732-R745 (2005).

14 Okuma M, Mochida J, Nishimura K, Sakabe K, Seiki K: Reinsertion of stimulated nucleus pulposus cells retards intervertebral disc degeneration: an in vitro and in vivo experimental study. J. Orthop. Res. 18(6), 988-997 (2000).

15 Gruber HE, Johnson TL, Leslie K et al.: Autologous intervertebral disc cell implantation: a model using Psammomys obesus, the sand rat. Spine 27(15), 1626-1633 (2002).

16 Nomura T, Mochida J, Okuma M, Nishimura K, Sakabe K: Nucleus pulposus allograft retards intervertebral disc degeneration. Clin. Orthop. Relat. Res. 389, 94-101 (2001).

17 Le Maitre CL, Freemont AJ, Hoyland JA: Accelerated cellular senescence in degenerate intervertebral discs: a possible role in the pathogenesis of intervertebral disc degeneration. Arthritis Res. Ther. 9(3), R45 (2007).

18 Roberts S, Evans EH, Kletsas D, Jaffray DC, Eisenstein SM: Senescence in human intervertebral discs. Eur. Spine J. 15 (Suppl. 3), S312-S316 (2006).

19 Le Maitre CL, Freemont AJ, Hoyland JA: Localization of degradative enzymes and their inhibitors in the degenerate human intervertebral disc. J. Pathol. 204(1), 47-54 (2004).

20 Pittenger MF, Mackay AM, Beck SC et al: Multilineage potential of adult human mesenchymal stem cells. Science 284(5411), 143-147 (1999).

21 Richardson SM, Hughes N, Hunt JA, Freemont AJ, Hoyland JA: Human mesenchymal stem cell differentiation to NP-like cells in chitosan-glycerophosphate hydrogels. Biomaterials 29(1), 85-93 (2008).

22 Risbud MV, Albert TJ, Guttapalli A et al.: Differentiation of mesenchymal stem cells towards a nucleus pulposus-like phenotype in vitro: implications for cell-based transplantation therapy. Spine 29(23), 2627-2632 (2004).

23 Crevensten G, Walsh AJ, Ananthakrishnan D et al: Intervertebral disc cell therapy for regeneration: mesenchymal stem cell implantation in rat intervertebral discs. Ann. Biomed. Eng. 32(3), 430-434 (2004).

24 Sakai D, Mochida J, Yamamoto Y et al:: Transplantation of mesenchymal stem cells embedded in Atelocollagen gel to the intervertebral disc: a potential therapeutic model for disc degeneration. Biomaterials 24(20), 3531-3541 (2003).
25 Richardson SM, Walker RV, Parker S et al.: Intervertebral disc cell-mediated mesenchymal stem cell differentiation. Stem Cells 24(3), 707-716 (2006).

26 Yamamoto Y, Mochida J, Sakai D et al.: Upregulation of the viability of nucleus pulposus cells by bone marrow-derived stromal cells: significance of direct cell-to-cell contact in coculture system. Spine 29(14), 1508-1514 (2004).

27 Vadala G, Studer RK, Sowa G et al.: Coculture of bone marrow mesenchymal stem cells and nucleus pulposus cells modulate gene expression profile without cell fusion. Spine 33(8), 870-876 (2008).

28 Sobajima S, Vadala G, Shimer A, Kim JS, Gilbertson LG, Kang JD: Feasibility of a stem cell therapy for intervertebral disc degeneration. Spine J. 8(6), 888-896 (2008).

29 Yang SH, Wu CC, Shih TT, Sun YH, Lin FH: In vitro study on interaction between human nucleus pulposus cells and mesenchymal stem cells through paracrine stimulation. Spine 33(18), 1951-1957 (2008).

30 Svanvik T, Barreto Henriksson H, Karlsson C, Hagman M, Lindahl A, Brisby H: Human disk cells from degenerated disks and mesenchymal stem cells in co-culture result in increased matrix production. Cells Tissues Organs 191(1), 2-11 (2010)

31 Lu ZF, Zandieh Doulabi B, Wuisman PI, Bank RA, Helder MN: Differentiation of adipose stem cells by nucleus pulposus cells: configuration effect. Biochem. Biophys. Res. Commun. 359(4), 991-996 (2007).

32 Livak KJ, Schmittgen TD: Analysis of relative gene expression data using real-time quantitative PCR and the 2(-Delta Delta C(T)) method. Methods 25(4), 402-408 (2001).

33 Henriksson HB, Svanvik T, Jonsson M et al: Transplantation of human mesenchymal stems cells into intervertebral discs in a xenogeneic porcine model. Spine (Phila Pa 1976) 34(2), 141-148 (2009).

34 Caplan AI, Dennis JE: Mesenchymal stem cells as trophic mediators. J. Cell Biochem. 98(5), 1076-1084 (2006).

35 Feng G, Wan Y, Balian G, Laurencin CT, Li X: Adenovirus-mediated expression of growth and differentiation factor- 5 promotes chondrogenesis of adipose stem cells. Growth Factors 26(3), 132-142 (2008).

36 Kawamura K, Chu CR, Sobajima $S$ et al. Adenoviral-mediated transfer of TGF- $\beta 1$ but not IGF-1 induces chondrogenic differentiation of human mesenchymal stem cells in pellet cultures. Exp. Hematol. 33(8), 865-872 (2005). 
Bai X, Xiao Z, Pan Y et al.: Cartilage-derived morphogenetic protein-1 promotes the differentiation of mesenchymal stem cells into chondrocytes. Biochem. Biophys. Res. Commun. 325(2), 453-460 (2004).

38 Steinert AF, Palmer GD, Pilapil C, Ulrich N, Evans $\mathrm{CH}$, Ghivizzani SC: Enhanced in vitro chondrogenesis of primary mesenchymal stem cells by combined gene transfer. Tissue Eng. Part A 15(5), 1127-1139 (2009).

Indrawattana $\mathrm{N}$, Chen $\mathrm{G}$, Tadokoro $\mathrm{M}$ et al.: Growth factor combination for chondrogenic induction from human mesenchymal stem cell. Biochem. Biophys. Res. Commun. 320 (3), 914-919 (2004).
Djouad F, Delorme B, Maurice M et al.: Microenvironmental changes during differentiation of mesenchymal stem cells towards chondrocytes. Arthritis Res. Ther. 9(2), R33 (2007).

41 Li X, Leo BM, Beck G, Balian G, Anderson GD: Collagen and proteoglycan abnormalities in the GDF-5-deficient mice and molecular changes when treating disk cells with recombinant growth factor. Spine 29(20), 2229-2234 (2004).
42 Sakai D, Mochida J, Iwashina T et al.: Regenerative effects of transplanting mesenchymal stem cells embedded in atelocollagen to the degenerated intervertebral disc. Biomaterials 27(3), 335-345 (2006).

43 Zhang YG, Guo X, Xu P, Kang LL, Li J: Bone mesenchymal stem cells transplanted into rabbit intervertebral discs can increase proteoglycans. Clin. Orthop. Relat. Res. 430, 219-226 (2005). 